\title{
GENERATOR FOR AUDIO CURRENTS OF ADJUSTABLE FREQUENCY WITH PIEZO-ELECTRIC STABILIZATION
}

\author{
By August Hund
}

ABSTRACT

A beat-frequency generator is described for producing audio currents which are practically sinusoidal. The frequency is adjustable and stabilized by means of a piezo-electric quartz disk and can be directly read off on a scale. The holder containing the piezo-electric element has a hand control for changing the frequency of the quartz disk by a small amount (a fraction of a cycle to several cycles).

On account of the piezo-electric control, it is possible to reset the calibration for the frequency for most any useful B voltage on the tubes producing the two high-frequency currents which beat with each other. The resetting can be carried on without any standard by means of the filament rheostat common to both oscillator tubes. The slow visible vibrations on the meter for the anode current of the piezo-electric oscillator are utilized for the resetting of the scale.

A thermostatic control is provided for very accurate work. Two filter detector circuits are described for obtaining audio currents of a good wave shape and keeping any high-frequency currents away from the load branch. A specially designed power amplifier is described. The same is used when more energy is required.

\section{CONTENTS}

I. Introduction Page

II. Method

III. Details _._. 633

IV. Operation of the generator. 636

V. Conclusions

\section{INTRODUCTION}

For electro-acoustic measurements it is advantageous to have a source generating alternating current within the audible range.

The research work carried on with piezo-electric crystals at the Bureau of Standards has shown that it is possible to produce audio currents with comparatively small ${ }^{1}$ quartz plates.

In this paper an arrangement is given for producing alternating currents of adjustable frequency and of a wave shape which is practically sinusoidal. A straight-line frequency condenser is used for changing the frequency within the audible range, and its scale is calibrated in audio frequencies. Smaller adjustments of frequency can be obtained by means of the effect of the crystal holder on the frequency of the piezo-electric element.

1 Proc. I. R. E., 14, p. 447; 1926. 


\section{METHOD}

In the arrangement of Figure 1 the outgoing audio current is produced by the interference of two high-frequency currents ${ }^{2}$ of frequencies $f_{1}$ and $f_{2}$, respectively. The frequency $f_{2}$ is fixed by the dimensions of a piezo-electric quartz disk and the position in its holder. It can be somewhat varied by raising or lowering the upper electrode in the holder. The variable frequency $f_{1}$ is adjusted by means of the variable air condenser $C_{2}$ which is in multiple with a fixed condenser $C_{1}$, the dial of the former being directly calibrated in audio-frequencies $\left(f_{1}-f_{2}\right)$. The finished apparatus is shown on

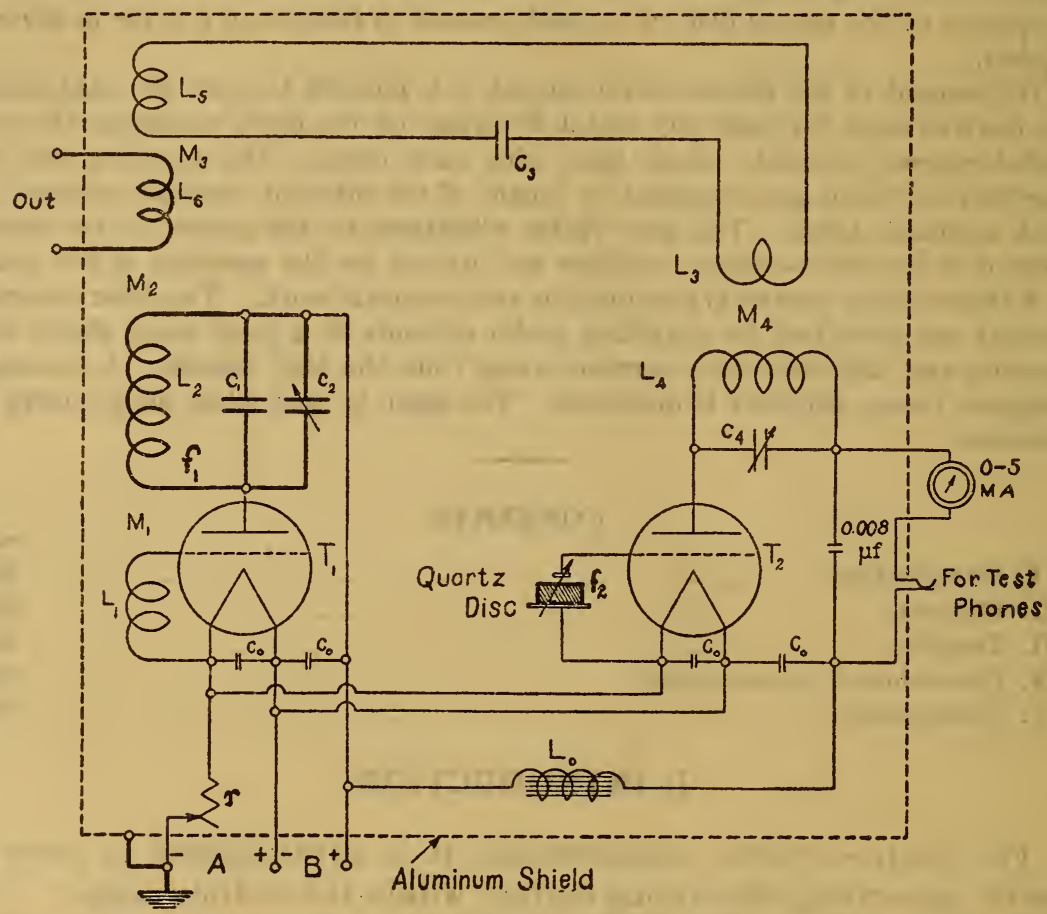

Fig. 1.-Beat-frequency generator with Piezo-electric control for producing audio currents of variable frequency

the photograph (fig. 2), together with a specially designed amplifier. The inside view is shown in Figure 3. The lettering on the coils and the essential condensers is the same as shown in Figure 1. The relative position of the coils which is shown in Figure 3 is of importance when a good wave shape of the audio current is desired.

2 Such a procedure has been used at the Bureau of Standards since 1923 and is based on the well-known beat principle. Circuits using the same principle, but not with piezo-electric stabilization, and small piezo-electric frequency adjustment as herein described, have been recently published in papers by B. S. Cohen, A. J. Aldridge, and K. West, J. I. E. E., 64, p. 1023; 1926; I. Wolff and A. Ringel, Proc. I. R. E., 15, p. 353; 1927; M. Gruetzenacher and E. Meyer, Elektrische Nachrichtentechnik, 4, p. 203; 1927. 
Scientific Papers of the Bureau of Standards, Vo!. 22

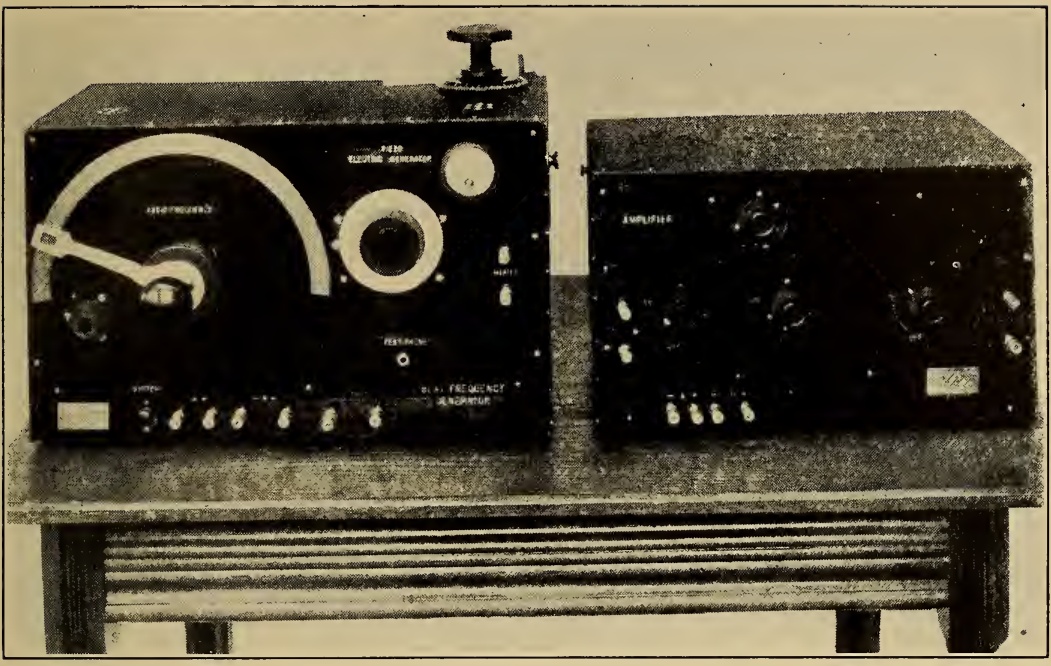

FIG. 2.-Generator and amplifier

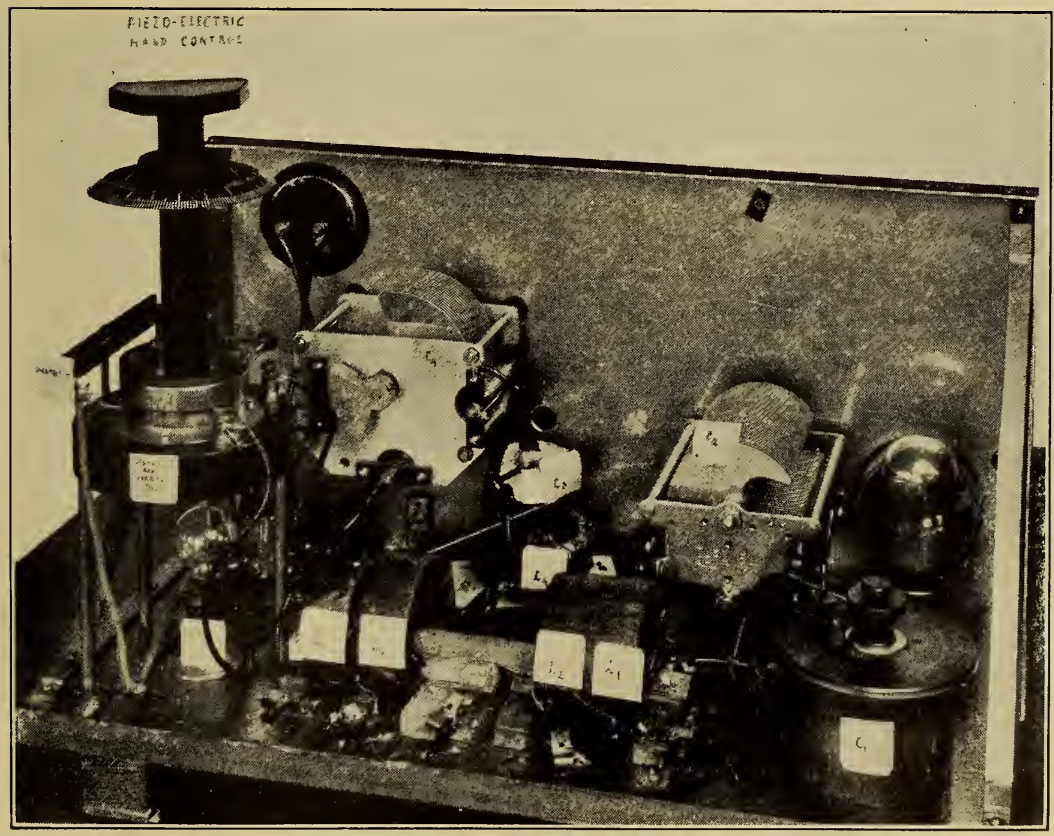

FIG. 3.-Inside view of the shielded generator 


\section{DETAILS}

The beat method has the inherent advantage that audio currents can be obtained by means of coils and condensers of comparatively small dimensions, their sizes being given by the respective radiofrequencies $f_{1}$ and $f_{2}$.

It has the disadvantage that any stray fields have to be prevented, and that one oscillator may have a frequency pull on the other oscillator as the component frequencies $f_{1}$ and $f_{2}$ approach each other. When the pulling effect on the frequency becomes very pronounced, it turns into a phase effect, so that for a certain variation of the air condenser $C_{2}$ in Figure 1 zero beat frequency $\left(f_{1}-f_{2}=0\right)$ would exist

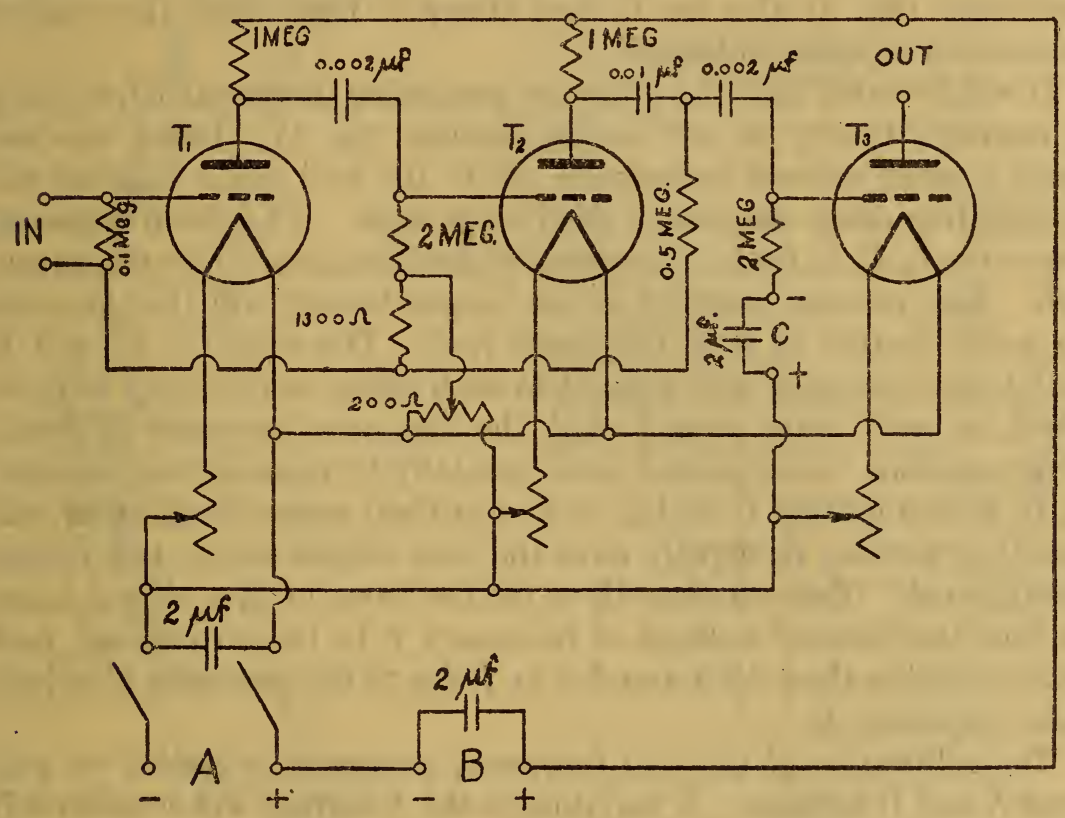

FIG. 4.-Power amplifier

and only a phase shift through $180^{\circ}$ would take place where $90^{\circ}$ represents the case of true synchronism of both high-frequency generators.

In the arrangement of Figure 1 the two generators producing the two high frequencies $f_{1}$ and $f_{2}$, respectively, are supplied by a common $\mathrm{A}$ and a common B battery. Both batteries serve also as sources for the amplifier when it is used and without noticeable frequency effects. It is necessary that the $\mathrm{A}$ as well as the B terminals of the beat-frequency generator (fig. 1) and the amplifier (fig. 4) are by-passed within the respective aluminum shieldings. A radiofrequency choke $L_{0}$ is also used between the two generators of the beat-frequency combination. The piezo-electric quartz disk has a 
diameter of about $48 \mathrm{~mm}$ and is about $7 \mathrm{~mm}$ thick. The lowest natural frequency of the disk is utilized. It is in the neighborhood of $64 \mathrm{kc} / \mathrm{sec}$. Since for the work for which the generator was designed a range from 0 to 4,000 cycles/second was sufficient, the values of $C_{1}$ and $L_{2}$ had to be chosen such that the scale of the air condenser $C_{2}\left(0.0007 \mathrm{mfd}\right.$. max. cap.) gave this range for $f_{1}-f_{2}$. The scale can not be clearly seen on the photograph of Figure 2 and is, therefore, given in more detail in Figure 5 . It will be noted that the scale is sufficiently open for practical use through its entire length. It is to be noted that another value for $C_{1}$ has to be chosen when the shielding is used, since the frequency $f_{1}$ is affected by it. For this reason it seems of advantage to use a variable air condenser (fig. 3) also for $C_{1}$ and clamp it tight after the original calibration is again obtained.

It will be noted that the generator producing the variable frequency $f_{1}$ employs tuning in the anode branch (fig. 1). Doing this and using a small mutual inductance $M_{1}$ to the grid turns $L_{1}$ gives rise to high-frequency currents of good wave form. The tuned step-over resonator $L_{3} C_{3} L_{5}$ feeds the current of fixed frequency $f_{2}$ to the output coil. Any rectifier inserted in the output branch will then produce an audio current in it of frequency $f_{1}-f_{2}$. The coils $L_{3}, L_{4}$, and $L_{1}$ and $L_{2}$ are arranged with respect to each other, so that they have no effect on each other except when the step-over resonator is closed. This condition was obtained experimentally by opening the resonator $L_{3} C_{3} L_{5}$ and placing $L_{3}$ and $L_{4}$ at first at right angles to all other coils and then turning $L_{3}$ slightly until the beat whistle in the test phones disappeared. The coupling $M_{4}$ to the few turns of $L_{3}$ is chosen loose, so that the induced voltage of frequency $f_{2}$ in the output coil $L_{6}$ is always smaller than the induced e. m. f. due to the generator of adjustable frequency, $f_{1}$.

The calibration of the beat-frequency generator is carried on with fixed $A$ and $B$ voltages. A variation in the A voltage will considerably affect the audio-frequency, since $f_{1}$ is changed. This gives, therefore, a means for resetting the calibration to that indicated on the dial. It can be done with a tuning fork, as follows: Suppose a 250-cycle fork is available. Then the pointer of condenser $C_{2}$ is set to the 250 mark on the dial and the filament resistance $r$ (fig. 1) varied until zero beat effect is noted between the sounds coming from the test phones and the fork. The resetting of the calibration is all the more accurate the lower the pitch of the standard sound. This leads to a still more simple resetting. The standard is omitted completely and the milliamperemeter in the anode branch of the piezo-electric generator used for noting zero beat condition between $f_{1}$ and $f_{2}$. The pointer of condenser $C_{2}$ is then set close to the zero mark corresponding to about 1 cycle/second and the resistance $r$ varied until the needle of the milliamperemeter shows vibrations of about one per second. 


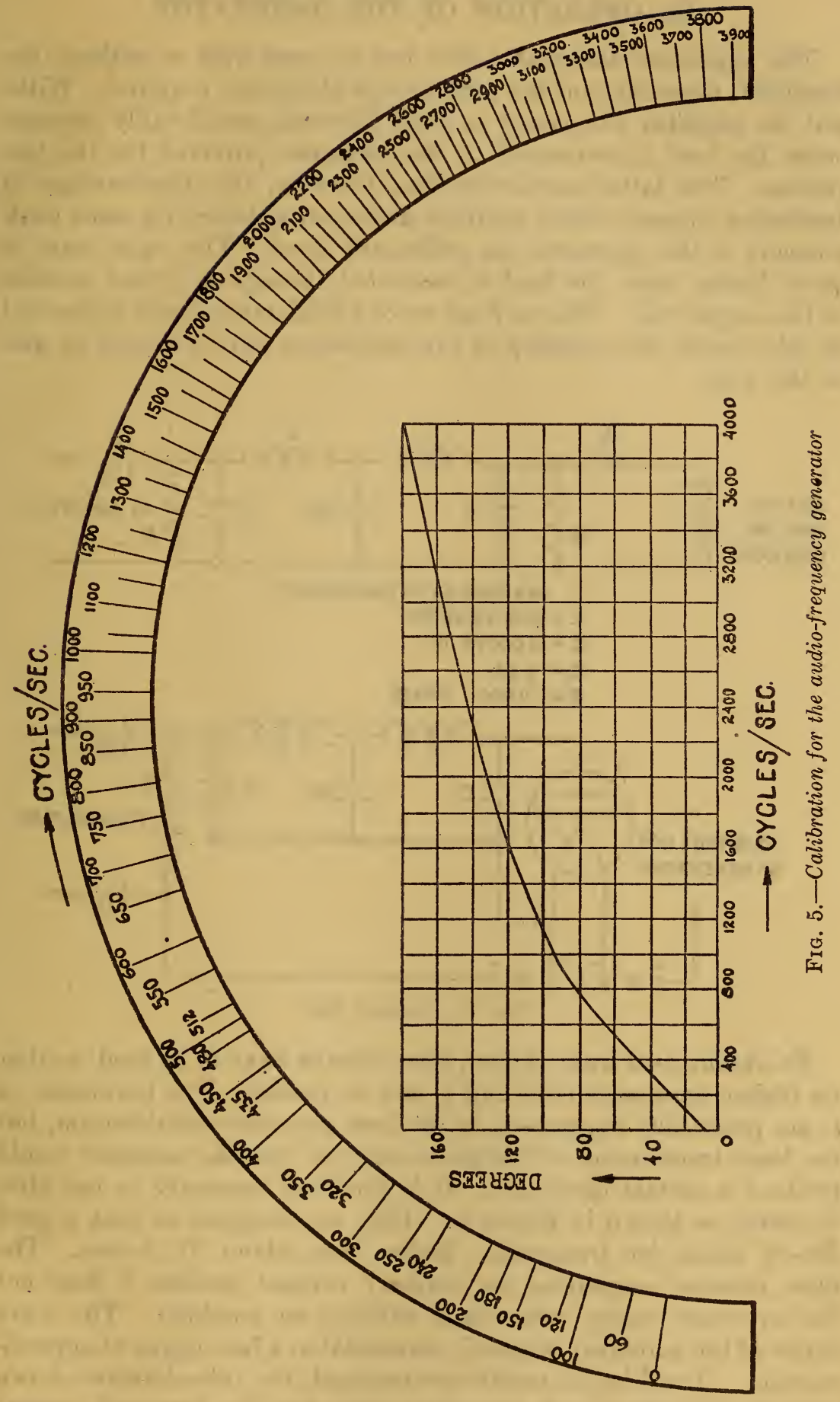




\section{OPERATION OF THE GENERATOR}

The apparatus described above can be used with or without the amplifier, depending on the purpose and the power required. Without the amplifier the energy is small although considerably stronger when the load is connected to the terminals provided for the test phones. The latter connection has, however, the disadvantage of producing currents which are more distorted, although for some work currents of this character are sufficiently good. The wave shape is much better when the load is connected through a crystal rectifier to the output coil. When a head set or a milliamperemeter is inserted in this circuit, the resetting of the calibration can be carried on also in this way.

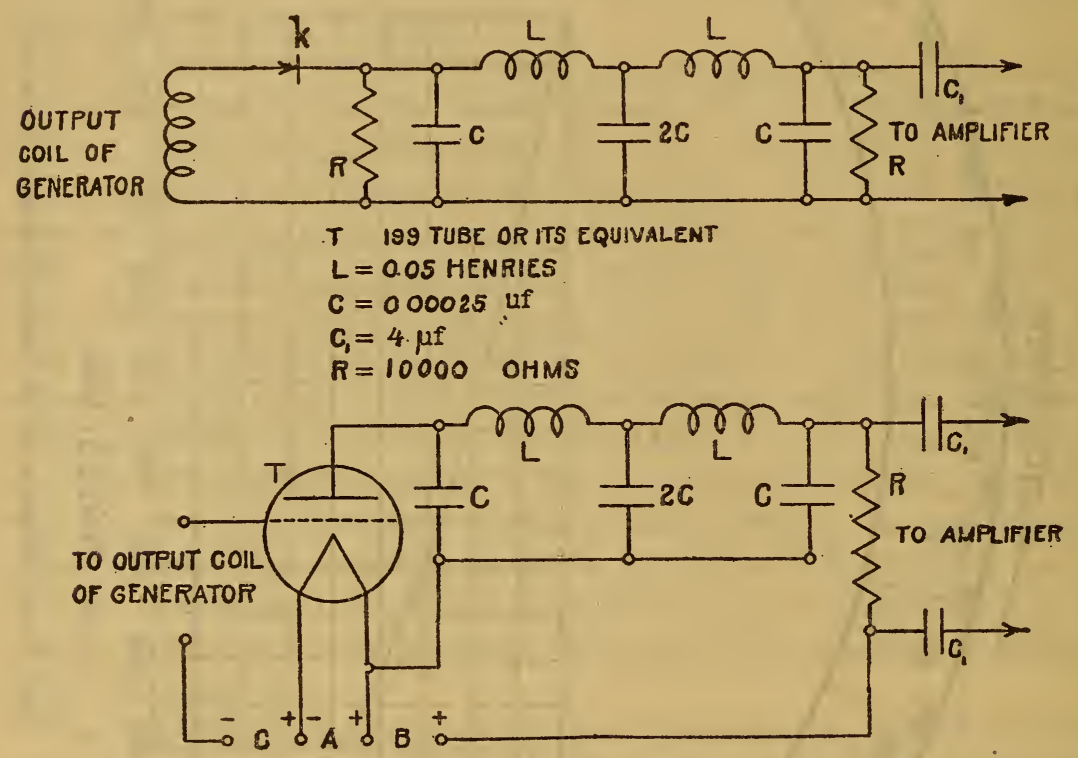

Fig. 6.-Detector filter

To obtain good wave shapes, filter circuits have to be used, so that no higher harmonics of $f_{1}$ and $f_{2}$ can be passed. The harmonics of $f_{2}$ are practically suppressed in the beat generator combination, but the least transference of harmonic currents into the amplifier would produce a certain distortion. It is therefore necessary to use filter detectors as shown in Figure 6. They are designed so that a good cut-off exists for frequencies higher than about $75 \mathrm{kc} / \mathrm{sec}$. The filter detector employing an ordinary contact rectifier $k$ does not deliver much energy when used without an amplifier. The wave shape of the amplified current is sinusoidal to a fair degree of approximation. If still better results are required, the tube detector shown in the same figure with a negative grid bias for zero grid current 



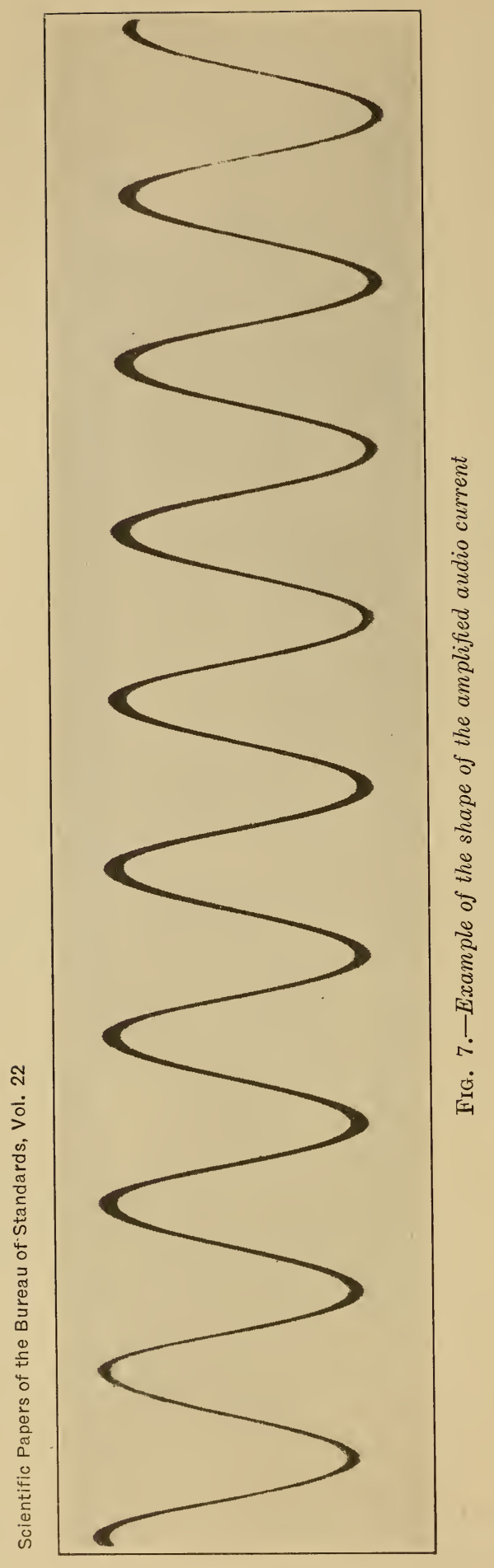


works satisfactorily. As an example, the oscillogram of a 70-cycle output current is shown in Figure 7. When no amplifier is needed, the coupling condensers to the amplifier can be dispensed with.

With the resetting of the calibration as described above, any frequency can be directly read on the dial. For very accurate work the scale can be calibrated by means of a harmonic method, and a thermostatic control is employed during the operation. It is shown in Figure 3 and consists of an incandescent lamp below the crystal holder and a thermostatic interrupter mounted in the top plate of the holder. The piezo-electric hand control gives a means to vary the audio frequency by a small amount (fraction of a cycle to several cycles).

\section{CONCLUSIONS}

A beat-frequency generator is described for producing audio currents of good wave shape and adjustable frequency. The arrangement is stabilized by means of a piezo-electric quartz disk, so that the adjustable audio frequency can be directly read off on a scale. The holder containing the piezo-electric element has a hand control for changing the frequency of the quartz disk by a small amount. A specially designed power amplifier and filter detectors are described.

Washington, September 27, 1927. 